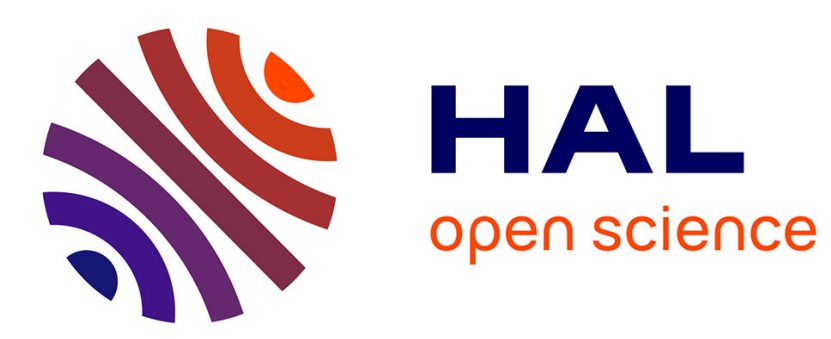

\title{
Real-time monitoring of cyclic nucleotide signaling in neurons using genetically encoded FRET probes.
}

Pierre Vincent, Nicolas Gervasi, Jin Zhang

\section{To cite this version:}

Pierre Vincent, Nicolas Gervasi, Jin Zhang. Real-time monitoring of cyclic nucleotide signaling in neurons using genetically encoded FRET probes.. Brain Cell Biol, 2008, 36 (1-4), pp.3-17. 10.1007/s11068-008-9035-6 . hal-00342452

HAL Id: hal-00342452

https://hal.science/hal-00342452

Submitted on 27 Nov 2008

HAL is a multi-disciplinary open access archive for the deposit and dissemination of scientific research documents, whether they are published or not. The documents may come from teaching and research institutions in France or abroad, or from public or private research centers.
L'archive ouverte pluridisciplinaire HAL, est destinée au dépôt et à la diffusion de documents scientifiques de niveau recherche, publiés ou non, émanant des établissements d'enseignement et de recherche français ou étrangers, des laboratoires publics ou privés. 


\title{
Real-time monitoring of cyclic nucleotide signaling in neurons using genetically- encoded FRET probes
}

\author{
Pierre VINCENT ${ }^{1,2}$, Nicolas GERVASI ${ }^{1,2}$ and Jin ZHANG $^{3}$ \\ 1: CNRS UMR 7102 Neurobiologie des Processus Adaptatifs, Paris. 9 quai St \\ Bernard, 75005 Paris, FRANCE \\ 2: UPMC UMR7102 Neurobiologie des Processus Adaptatifs, Paris. 9 quai St \\ Bernard, 75005 Paris, FRANCE \\ 3: Department of Pharmacology and Molecular Sciences, Solomon H. Snyder \\ Department of Neuroscience and Department of Oncology, The Johns Hopkins \\ University School of Medicine, Baltimore, Maryland 21205, USA. \\ pierre.vincent@upmc.fr; jzhang32@jhmi.edu
}

\begin{abstract}
The signaling cascades involving cyclic nucleotides play a key role in signal transduction in virtually all cell types. Elucidation of the spatiotemporal regulation of cyclic nucleotide signaling requires methods for tracking the dynamics of cyclic nucleotides and the activities of their regulators and effectors in the native biological context. Here we review a series of genetically encoded FRET-based probes for real time-monitoring of cyclic nucleotide signaling with a particular focus on their implementation in neurons. Current data indicate that neurons have a very active metabolism in cyclic-nucleotide signaling, which is tightly regulated through a variety of homeostatic regulations.

Cyclic nucleotides; fluorescence imaging

Imaging cyclic nucleotides in neurons

\section{Introduction}

A number of neuropsychiatric pathologies are related to impairments of neuromodulatory systems, as demonstrated by the number of drugs used in clinics which target neuromodulators, acting either on reuptake systems or on G-protein coupled receptors. Among the intracellular signaling cascades triggered by neuromodulators, signaling pathways regulated by cyclic adenosine monophosphate (cAMP) and cyclic guanosine monophosphate (cGMP) are known to be responsible for the modulation of many cellular functions in the brain. cAMP, as the prototype second messenger, plays important roles in a wide array 
of neuronal functions including specific forms of synaptic plasticity, cognitive functions, neuronal survival and axonal regeneration. Many of these responses are mediated by cAMP-dependent protein kinase (PKA), which has served as a model of protein kinase structure and regulation. On the other hand, the exchange protein directly activated by cAMP (Epac) and cAMP-regulated channels are also important cAMP effectors. cGMP is another intracellular second messenger that plays an important signaling role in neurons, particularly in mediating the response to the gas neuromodulator nitric oxide.

Spatial compartmentation of cyclic nucleotide action at the subcellular level was first suggested more than 20 years ago, and recent studies provide new evidence that a general increase in cyclic nucleotide concentration in the cell is not sufficient for the specific regulation of functional effects. For generating appropriate cell responses, precise spatial and temporal control of cyclic nucleotide signaling must be achieved. In order to better understand cyclic nucleotide actions within their signaling context, methods are needed to analyze their dynamics in living cells. Ideally such methods should provide high spatial and temporal resolution for tracking the activity changes continuously and for following them in different compartments and microdomains. Furthermore, quantitative information should be obtained from individual cells, because some important aspects of signal transduction, such as transient and oscillatory activity, can be averaged out during studies of a population of cells. Care must also be taken to ensure that the signal is specific, and that expression of the probe does not perturb the phenomenon that the probe is supposed to report. A major breakthrough in the study of cyclic-nucleotide mediated intracellular signaling has arisen with the new optical biosensors that have been developed since 2001. Genetically encoded probes used to monitor the cAMP/PKA cascade have been reviewed recently (Willoughby and Cooper, 2008; Lohse et al., 2008) and we focus here on applications of FRET-based biosensors that are capable of tracking cyclic nucleotide signaling in living cells with a particular focus on their implementation in neurons.

\section{Cyclic AMP and PKA}

The first probe constructed to report changes in cAMP concentration was FlCRhR (Adams et al., 1991). This probe used chemically labeled PKA subunits 
whose dissociation was monitored by FRET. The FlCRhR probe was used on some favorable preparations like large invertebrate neurons (Bacskai et al., 1993; Hempel et al., 1996) or Xenopus embryonic neurons (Gorbunova and Spitzer, 2002); however, its use in mature vertebrate neurons was limited by the difficult task of introducing the probe as an intact holoenzyme into the cytosol (Vincent and Brusciano, 2001). Various gene transfer methods now allow the expression of genetically-encoded probes in virtually any cell type including neurons, and probes using CFP and YFP as fluorophores were soon derived from the previous FlCRhR (Zaccolo et al., 2000; Lissandron et al., 2005). This assay depends on the coexpression of two genes coding for the two moieties of the probe at a similar level, a condition that must be achieved for proper functioning of the probe. Unfortunately, these PKA-derived probes have an intact kinase domain, so the presence of the probe inherently increases PKA activity inside the cell. This has been reported with FlCRhR in cardiomyocytes where probe diffusion into the cell partially activated L-type calcium channels (Goaillard et al., 2001). Neurons have a tonic cAMP production and PKA activity; the introduction of a large load of extra PKA inside the neuron will likely perturb this equilibrium, first by buffering a large part of intracellular cAMP (this in itself may not be a problem) and second by releasing an active PKA subunit that will impact feed-back mechanisms. The PKA-derived probes also suffer from kinetic limitations (see below). Despite all these limitations, the genetically-encoded PKA-based probe has been successfully used on cardiocytes where it reported changes in PKA dissociation state in response to beta-adrenergic stimulation (Mongillo et al., 2004), and it may prove useful in some specific neuronal applications, particularly where a highly sensitive qualitative monitoring is needed. For example, a PKA-based genetically encoded sensor reported responses to activators of the PKA cascade in transgenic Drosophila, although probe expression led to severe developmental defects (Lissandron et al., 2007).

Besides PKA, one important target of intracellular cAMP is a family of cyclic-nucleotide gated channels, one of its members being expressed in olfactory neurons. The sensitivity of these membrane channels to cAMP was used to measure changes in sub-membrane cAMP concentration either by electrophysiological recordings or by imaging the calcium influx through these channels (Rich et al., 2001a). This elegant approach showed that the dynamics of 
cAMP differ at the membrane and in the cytosol (Rich et al., 2001b). Unfortunately, these channels cannot be expressed in neurons without profoundly affecting their electrophysiological properties. Moreover, as these channels are permeable to calcium, their activation would increase local intracellular calcium level which may affect the cAMP cascade. Further work along this line may eventually lead to reporter channels that may have limited impact on neuronal excitability.

Cyclic AMP directly modulates another important membrane current in neurons, $\mathrm{I}_{\mathrm{h}}$, which is involved in many physiological activities such as the control of the resting membrane potential, rhythmic activity, and integration of synaptic currents (Kaupp and Seifert, 2001). The HCN2 channel contributes to the $\mathrm{I}_{\mathrm{h}}$ current, and its intracellular cAMP binding domain was used to create a cytosolic probe, called HCN2-camps. This probe, as most of the probes presented below, is built on the now classical pattern of a domain sensitive to the signal of interest sandwiched between CFP and YFP fluorophores. When cAMP binds to HCN2camps binding domain, the conformational change that follows decreases FRET between CFP and YFP with an $\mathrm{EC}_{50}$ of $6 \mu \mathrm{M}$. This probe proved suitable to monitor changes in cAMP levels in cardiomyocytes (Nikolaev et al., 2006a) and may be very useful in neurons as well.

Other genetically-encoded fluorescent FRET sensors were derived from another protein family, Epac, a cAMP receptor protein mediating guanine nucleotide exchange activity toward a small guanosine triphosphatase, Rap1. The probe ICUE-1 was constructed from the full-length Epac sequence (DiPilato et al., 2004). ICUE2 (Dunn et al., 2006; Violin et al., 2008) shares its configuration (figure 1A) with another probe called CFP-Epac(SDEP-CD)-YFP (Ponsioen et al., 2004) that contains Epac1 with a deletion of the N-terminal domain for reducing the attachment to intracellular membrane. Further deletion of Epac keeping only the cAMP-binding domain generated a smaller sensor called Epac1-camps (Nikolaev et al., 2004). Epac has a lower sensitivity to cAMP than PKA, and dose-response curves for this family of probes indicate sensitivities in the micromolar range.

In addition to directly measuring cAMP concentration, the downstream biochemical effect of PKA on protein phosphorylation can also be monitored. A- 
kinase activity reporter (AKAR) is a recombinant protein composed of a phosphoamino acid binding domain and PKA-specific substrate sandwiched between CFP and YFP (figure 1B). When phosphorylated by PKA, intramolecular binding of the substrate by the phosphoamino acid binding domain drives a conformational reorganization, leading to an increase in FRET between CFP and YFP. The FRET response of the first probes in this class, AKAR1 (Zhang et al., 2001), was largely irreversible in living cells. It was hypothesized that tight binding of the phosphorylated substrate to the phosphoaminoacid binding domain (which was the 14-3-3 protein) can prevent the phosphorylated substrate from being dephosphorylated by phosphatases and lead to apparent irreversibility of the reporter. In order to continuously monitor the dynamic balance between the kinase and phosphatase activities, however, it is desirable that the kinase activity reporter be reversible. The replacement of the 14-3-3 with FHA1, a modular phosphothreonine binding domain with a weaker binding affinity than that of 14-3-3, led to the generation of AKAR2, a reversible AKAR reporter (Zhang et al., 2005). Additionally, use of GFP variants with a reduced tendency to dimerize (Zacharias et al., 2002) as FRET donor and acceptor in AKAR2.2 helped to improve reversibility (Dunn et al., 2006). In the most recent efforts of sensor optimization, using circular permutants of fluorescent protein to replace wild-type YFP as the FRET acceptor led to improved dynamic range, presumably via changes of the relative orientation of the donor and acceptor fluorophores. AKAR3, the current version of AKAR, utilized a circularly permutated Venus (cpV E172) and doubled the response amplitude of AKAR2 (Allen and Zhang, 2006).

AKAR probes exhibit a FRET increase in response to forskolin (a drug which activates adenylyl-cyclases), and this effect was observed with AKAR2 in cortex, intralaminar thalamic neurons (Gervasi et al., 2007) and ventrobasal thalamus (Gervasi and Vincent, unpublished). AKAR2 also responds to neuromodulators that are positively coupled to the cAMP/PKA cascade, such as serotonin via the 5$\mathrm{HT}_{7}$ receptor in thalamic intralaminar neurons (Gervasi et al., 2007). It is important to note that the probe responds to low concentrations of neuromodulators (100 $\mathrm{nM} 5-\mathrm{CT}$ to activate $5-\mathrm{HT}_{7}$ receptors), while neither phosphatases nor phosphodiesterases were blocked. AKAR2 thus has a high sensitivity to report the downstream effect, at the kinase level, of a 
neuromodulatory signal received by a neuron.

Whether the recorded signal depends on phosphorylation must be verified using a mutant of AKAR2 where the phosphorylated threonine is mutated to an alanine (AKAR2mut). Indeed, several artifacts may affect the fluorescence ratio by differentially affecting the optical properties of the pair of fluorophores. The negative control mutant was tested in several preparations to verify that the measured signal reflected a genuine phosphorylation in the PKA-specific site. As expected, agonists or drugs known to activate PKA in the cortex or in intralaminar thalamic nucleus such as forskolin, dopamine, serotonin, and 5- $\mathrm{HT}_{7}$ agonists, all failed to induce any fluorescence ratio change with AKAR2mut (Gervasi et al., 2007 and unpublished data).

While the fluorescence change has thus been shown to depend on phosphorylation, the specific involvement of PKA still needs to be evaluated. First, the phosphorylation site of the probe was designed to be specific for PKA. The substrate sequence is based on the specific PKA substrate kemptide (Kemp, 1980) and consistent with PKA consensus site of $R_{-3}-R-x_{-1}-S / T-B_{+1}$, where $R$ is arginine, $\mathrm{x}$ refers to any amino acid, $\mathrm{B}$ to a hydrophobic residue, and the $\mathrm{S}$ or $\mathrm{T}$ to the site of phosphorylation (Zetterqvist et al., 1976; Smith et al., 1999). Then, several kinases that have overlapping substrate preferences, such as PKC, CaMKII, PKG, were tested and found to have no major effects on AKAR (Zhang et al., 2001). Finally, it is important to use a specific blocker of PKA to demonstrate that this kinase is indeed responsible for the fluorescence change. In brain slices, PKA was blocked using whole-cell recording with $500 \mu \mathrm{M}_{P^{-}}$ cAMPS in the pipette, which blocked the response mediated by the $5-\mathrm{HT}_{7}$ receptors (Gervasi et al., 2007).

While the consensus phosphorylation site has been designed to be specific for PKA, several phosphatase types can theoretically dephosphorylate the probe. In the cortex, cantharidin and cyclosporin A which together inhibited protein phosphatases $1,2 \mathrm{~A}$ and $2 \mathrm{~B}$, were shown to prevent the dephosphorylation of AKAR2 (Gervasi et al., 2007). A more specific pharmacology is now needed to identify the phosphatase(s) involved in this dephosphorylation. Once these phosphatases are identified, the recovery of AKAR2 ratio could be used to monitor phosphatase activities. 


\section{Cyclic GMP}

Nitric oxide is an unconventional neuromodulator which diffuses across membranes and acts principally by activating cGMP synthesis by the soluble guanylyl cyclase. Various probes have been designed in order to report either the release of NO or the production of cGMP. Despite the considerable importance of NO in neuroscience, few of these probes have been actually used in neurons.

A NO-sensitive probe was designed by fusing the heme-binding domain of guanylyl cyclase with EGFP. This probe, named HBR-GFP, responds to NO by an increase of GFP fluorescence, which can be directly monitored in cells, and a change in the excitation spectrum of the probe, which might be monitored ratiometrically by dual wavelength excitation. EGFP was used as a control to verify that the effects depended on the heme-binding moiety of the protein, and pharmacological blockade of NO synthase confirmed that the recorded signals depended on NO. The onset of the response to NO is in the range of hundred of seconds, making this probe unsuitable to monitor rapid neuronal events. Dissociation of NO from the probe is even slower, so the probe only reports the cumulative release of NO. This probe was used in cerebellum slices to evaluate the radial spread of NO within the cerebellar cortex, showing that NO release as a function of parallel fiber stimulation exhibits an unexpected bell-shaped curve (Namiki et al., 2005).

Downstream of NO, cGMP can be measured directly using PKG as a sensor. These probes named CGY (Sato et al., 2000) and Cygnet-2 (Honda et al., 2001) faithfully report changes in intracellular cGMP concentration (figure 1C). Interestingly, although these probes have similar structure and fluorophores, the FRET changes in response to cGMP are in opposite directions. While the selectivity of CGY for cGMP against cAMP is debated (Nikolaev et al., 2006b), Cygnet-2 is clearly insensitive to physiologically-relevant cAMP concentrations and has a Kd for cGMP around $1 \mu \mathrm{M}$ (Honda et al., 2005; Nikolaev et al., 2006b). It is important to note that the kinase domain from PKG is inactivated by mutation in the Cygnet-2 probe so as not to phosphorylate proteins that may be involved in this signaling cascade. The CGY sensor has been further modified by fusing it to guanylyl cyclase, thus creating a probe sensitive to low NO concentrations (Sato et al., 2005). This may be useful in neuronal preparations to detect very low NO 
concentrations. It has been shown, however, that resting NO concentrations are sufficient to elevate intracellular cGMP to a level that can be detected with Cygnet-2 (Hepp et al., 2007).

New probes using cGMP-binding domains from PKG or phosphodiesterases have been constructed recently and display faster and/or larger responses. These promising tools have not been tried on neurons yet (Nikolaev et al., 2006b; Russwurm et al., 2007; Nausch et al., 2008).

\section{Quantification}

Probes relying only on a change in fluorescence intensity at one wavelength are inherently more sensitive to various artifactual changes such as instrumental fluctuations, cell movements or changes in probe concentration. FRET probes remedy these problems by producing an opposite change in fluorescence intensity at two different wavelengths, which can be monitored using intensity-based methods. Another detection method called "fluorescence life-time imaging" (FLIM) monitors FRET by the changes in life-time of the donor excited state and this method is also independent on the fluorophore concentration. The various quantification approaches have their specific advantages and drawbacks, as has been described for test CFP-YFP fusion constructs (Domingo et al., 2007). We review here the currently used methods that may be useful for neuronal preparations.

Ratiometric quantification is the most straightforward method, relying on a single excitation wavelength centered on $436 \mathrm{~nm}$ for CFP and dual detection at 480 (F480) and $530 \mathrm{~nm}$ (F530) for CFP and YFP, respectively, with each image being background corrected. The F530/F480 ratio cancels out artifactual changes in intensity and this ratio is monitored continuously during the experiment as an indicator of FRET changes.

Ratiometric quantification as well as the other intensity-based quantifications strongly depend on an accurate background subtraction. This background cannot be measured directly, since no reference image can be obtained before the probe is expressed. The background value is usually assumed to be a constant value for the whole image, estimated by measuring the autofluorescence in a region of the preparation where no fluorescent probe is expressed. Errors in background 
subtraction can lead to errors in absolute ratio values, and the lesser the fluorescence intensity of the probe, the bigger the error. This may be particularly important when comparing different cells or different cellular domains with different brightness. Indeed, comparisons of small neuronal compartments such as dendritic trees, spines or axon terminals with the much brighter soma require a careful background correction and experimental confirmation with the control inactive probe. When measuring the average ratio over a cellular structure with various intensity levels, dim pixels corresponding to regions of low probe concentration bring a less precise estimate of the ratio value compared to bright pixel where the probe gives a better signal/noise ratio. Dim pixels are usually removed using a fixed (and somewhat arbitrary) threshold. A more objective quantification uses all pixels in the region of interest and calculates an average ratio with each pixel weighted by its intensity (Tsien and Harootunian, 1990).

Another error would result from time-dependent changes in background value which would produce a continuous drift of the ratio during the experiment. In practice, all these errors are minimized by recording only cells (or regions of a cell) where the fluorescence intensity is much larger (at least 3 times more) than the autofluorescence. At the other extreme, too high an expression of the probe may lead to probe aggregation or cause a cell to be stressed by too much protein expression, so that a good compromise should be determined and validated by biological controls.

Quantification errors may also stem from the probe itself. For example, AKAR2 targeted to the nucleus using an NLS signal exhibited a lower average ratio than AKAR2 in the cytosol. This difference was also observed with AKAR2mut, showing that this effect did not reflect a difference in basal PKA activity level but rather indicated that the biophysical environment in the nucleus must be different from the cytosol and differentially affect the CFP-YFP interaction. The molecular basis of this effect remains to be determined.

These factors contribute to some cell-to-cell dispersion in the basal ratio value, which is observed even with the insensitive AKAR2mut probe. As a consequence, an absolute calibration of the ratio cannot be performed unless the minimal ratio value $\left(R_{\min }\right)$ and the maximal ratio value $\left(R_{\max }\right)$ is measured for each cell in the preparation. Concerning AKAR2, $R_{\max }$ is the ratio when all of the 
probe molecules are phosphorylated: this value can be easily measured by adding forskolin at the end of the recording. This drug strongly activates adenylyl cyclases and increases the ratio to a level that is not further increased either by phosphodiesterases (Gervasi et al., 2007) or by phosphatase inhibitors (data not shown), showing that the probe is saturated. This ratio value can thus be considered as equal to $\mathrm{R}_{\max }$. But $\mathrm{R}_{\min }$ is difficult to determine experimentally for AKAR2: phosphatases should be strongly activated while PKA is blocked, a situation difficult to achieve in living cells. Quantification with ratiometric imaging of AKAR2 therefore remains limited to measurements of responses as a percentage of the response to forskolin.

Quantification could be pushed a bit further with the cGMP-sensitive probe Cygnet-2 thanks to better pharmacological tools (Hepp et al., 2007). Increasing doses of a NO donor produced increasing ratio responses up to a maximal value for $50 \mu \mathrm{M}$ DEANO or $100 \mu \mathrm{M}$ SNAP. This level was not further increased by blockers of phosphodiesterases, thus representing $\mathrm{R}_{\max }$. Guanylyl cyclase could be rapidly and efficiently blocked by $10 \mu \mathrm{M}$ ODQ which, together with the strong endogenous phosphodiesterase activity, rapidly led to a ratio level significantly lower than baseline (Hepp et al., 2007). This level can be reasonably assumed to be close to $\mathrm{R}_{\min }$.

When the sensor behaves as a single ligand binding domain, the equations used for ratiometric calcium probes (Grynkiewicz et al., 1985) also apply to cAMP or cGMP:

$$
[\mathrm{cXMP}]=\beta \cdot \mathrm{K}_{\mathrm{d}} \cdot\left(\mathrm{R}-\mathrm{R}_{\min }\right) /\left(\mathrm{R}_{\max }-\mathrm{R}_{\min }\right) .
$$

In this equation, $R_{\min }$ is the ratio measured in the absence of ligand, $R_{\max }$ is the ratio when the probe is saturated with the ligand. $\beta . \mathrm{Kd}$ (apparent $\mathrm{Kd}$ ) depends on the imaging setup and can be determined experimentally on cell extracts. This type of quantification has been applied to cultured HEK293 cells expressing ICUE2, assuming that the resting cAMP concentration was well below $\mathrm{Kd}$ (i.e. resting ratio equals $R_{\min }$ ), and $R_{\max }$ was the ratio in the presence of forskolin (Violin et al., 2008).

CFP and YFP are far from perfect fluorophores, and their excitation and emission spectra overlap to some extent. One simple correction involves removing the part of CFP emission "spillover" or "bleed-through" collected at the YFP wavelength 
(Dunn et al., 2006; Shafer et al., 2008). However, it is not clear how this correction would improve the signal/noise ratio as it does not provide any additional data from the preparation but merely changes the ratio scale. A more sophisticated method involves the acquisition of a third fluorescence image with excitation of the acceptor and measurement of the acceptor fluorescence $(480 \mathrm{~nm}$ excitation and $530 \mathrm{~nm}$ emission for the CFP/YFP pair). This additional image provides an additional data element directly indicative of the acceptor concentration. These three images are processed through various calculation methods, commonly referred to as "three filter cube method" (Gordon et al., 1998; Hoppe et al., 2002; Zimmermann et al., 2002; Gu et al., 2004), and should provide more accurate estimates of FRET changes. However, these quantifications depend on a careful calibration of the imaging system, and the propagation of errors in the calculation resulting from inaccurate background subtraction or errors in calibration has not been studied carefully.

Acceptor photobleaching is a classical way to estimate FRET level; however, this destructive method could only be used at the end of a recording, and may thus complement the 3 filter cube method.

Ultimately, absolute FRET quantification would be obtained from the fluorescence lifetime of the FRET donor: the lifetime of the excited state of the donor decreases if the energy is transferred to the acceptor by FRET. This parameter is independent of the probe concentration and is directly related to FRET efficacy. Fluorescence lifetime imaging necessitates a dedicated imaging equipment working with nanosecond resolution. Recordings in the frequency domain are fast enough to report events pertaining to signal integration in neurons, and our preliminary recordings show that this method is indeed promising.

\section{Buffering effects}

Whether the expression of a probe perturbs the signal to be measured is an important question. We have tested this in detail with AKAR2 in cortical and thalamic brain slices. First, we verified that the expression of AKAR2 did not overtly affect neuronal properties: whole-cell patch-clamp recording showed that neurons expressing AKAR2 display the expected firing behavior in response to depolarizing or hyperpolarizing current injection (figure 2). Then, does a 
large intracellular concentration of AKAR2, by constituting an extra load of PKA substrate, "buffer" the kinase activity of PKA? This appears unlikely to cause major disturbance, since the kinase turnover rate is relatively fast (Gibbs et al., 1992) and PKA can phosphorylate a large number of endogenous proteins. This was confirmed experimentally in intralaminar thalamic neurons where $\mathrm{I}_{\mathrm{SAHP}}$, a PKA-sensitive potassium current, was as efficiently blocked by PKA in neurons expressing AKAR2 as in control neurons, showing that probe expression did not change the efficacy of endogenous PKA (Gervasi et al., 2007).

The situation may be different with biosensors that bind cyclic nucleotides, since the sensor is present at several tens of micromolar concentration in the cytosol and may thus strongly buffer any response. A similar buffering problem was encountered when intracellular signaling with the calcium ion was studied in neurons using chemical dyes, but with two main differences: 1) for each open calcium channel, thousands of calcium ions enter the cell per second instead of a few tens of cyclic nucleotide molecules being produced by a cyclase during the same time; 2) calcium ions are heavily buffered by a number of endogenous proteins present in neurons while no buffer seems to exist for cyclic nucleotides. As a consequence, when a chemical calcium dye is present at micromolar concentrations, a level similar or lower to the concentration of endogenous calcium-binding proteins, the calcium influx can be adequately detected optically without too much buffering artifact introduced by the dye. Besides, the diffusible dye actually speeds up the diffusion of calcium inside the cell. In contrast, binding of cyclic nucleotides to a large amount of slower-diffusing biosensor molecules could dampen and/or slow down changes in cyclic nucleotide concentration. If the cells synthesized a small and fixed amount of cyclic nucleotides in response to a stimulation, these molecules would activate a small percentage of the probe and would produce very small ratio changes. These predictions are not confirmed by current data since responses to neuromodulators or to calcium waves can be a large fraction of $\mathrm{R}_{\max }$ (Nikolaev et al., 2004; Dunn et al., 2006; Shafer et al., 2008), showing that neurons are certainly able to load a large fraction of the probe with cyclic nucleotides. Moreover, the buffering effect of the probe could be tested functionally in Drosophila: transgenic flies expressing the Epac1-camps probe in neurons exhibited no major perturbation in their daily locomotor activity, an indicator which is sensitive to changes in the cAMP cascade (Shafer et al., 
2008). The final cyclic nucleotide concentration must then proceed from a tightly controlled dynamic equilibrium in which cyclases produce a large number of cyclic nucleotide molecules until negative feed-back mechanisms become powerful enough to set a new steady-state level or initiate the recovery.

\section{Dynamic equilibrium of signaling cascades}

This is exemplified by thalamic neurons studied in brain slices where a tonic NO production maintains a continuous production of cGMP which could be detected by the Cygnet probe (Hepp et al., 2007). Tonic phosphodiesterase activity keeps the cGMP level constant at an intermediate level; pharmacological tools revealed the strong involvement of type II phosphodiesterase in this cGMP balance (Hepp et al., 2007). From this level, the cGMP concentration can either increase or decrease, depending on the up- or down-regulation of guanylyl cyclase and/or phosphodiesterase. Guanylyl cyclases and phosphodiesterases are thus powerful enzymes whose relative activities set the equilibrium point where the cGMP concentration stabilizes. The buffering effect of Cygnet probably has no effect on this final level but only slows changes from this equilibrium level.

A dynamic equilibrium is also seen in the cAMP/PKA cascade: when phosphodiesterases are blocked with IBMX, the AKAR2 ratio strongly increases (figure 3) indicating a tonic adenylyl cyclase activity which is continuously counteracted by phosphodiesterases. In Drosophila, cAMP imaging using the Epac1-camps sensor revealed that while most neurons studied in this preparation responded to neuropeptides by a FRET decrease, indicative of an increase in intracellular cAMP concentration, a fraction of the large lateral neurons occasionally responded to the DH31 peptide by a FRET increase, indicative of a decrease in cAMP concentration, i.e. a downward modulation of a tonic cAMP level (Shafer et al., 2008).

The tonic production of cAMP is sufficient to slightly activate PKA since, in cortical brain slices, the application of phosphatase inhibitors also increases the baseline AKAR2 ratio (Gervasi et al., 2007): the tonic PKA activity is reversed by phosphatases to maintain a steady-state level. Indeed, if AKAR2 was not continuously dephosphorylated, the low basal PKA activity would progressively lead to a maximal phosphorylation of the probe and no further response would be 
observed from this $\mathrm{R}_{\max }$ level. Fortunately, experiments show that baseline ratio is well below $\mathrm{R}_{\max }$, and AKAR2 responses to receptor stimulations still remain below this $\mathrm{R}_{\max }$ value: maximal stimulation of $5-\mathrm{HT}_{7}$ receptors increases the ratio value only to half the response to forskolin (Gervasi et al., 2007) and various

neuromodulators in the cortex produce graded ratio responses (figure 4). AKAR2 should thus be viewed as an indicator of the PKA/phosphatase balance with a sensitivity well suited to report neuromodulatory responses in neurons.

In a review, Houslay and Milligan described some cell types that have low "resting" cascade activity while other cell types display a high turnover of the cascade in a futile cycle (Houslay and Milligan, 1997). This has been later demonstrated using the highly-sensitive cyclic-nucleotide gated channel (C460W/E583M mutation): IBMX has no effect on baseline cAMP concentration on HEK293 cells, while the excitable pituitary-derived cell line GH4C1 shows a large response to IBMX (Rich et al., 2001a). Apparently, the neuronal types so far studied in rodent brain slices and Drosophila all belong to the "tonically-active" type for both cAMP and cGMP signaling cascades. Why would neurons benefit from a tonically active signaling cascades? One hypothesis is that a tonically active system allows for much wider range of signal integration than a simple on/off system, as modulation of any of the enzymes in the cascade can produce subtle changes in the steady-state level of the signal. It may also allow for faster responses if both synthesis is increased and degradation is decreased in a coordinated way.

\section{Kinetics of cAMP/PKA events}

The kinetics of a probe largely determine its temporal resolution for monitoring the cellular events. Based on the kinetics of purified PKA enzyme, the PKA-based probes have been suggested to dissociate slowly at low cAMP concentrations (Rich and Karpen, 2002) and would therefore be unsuitable to monitor rapid changes in cAMP concentration. This has been measured with the genetically-encoded cAMP-sensitive probe derived from PKA, which responds to cAMP with a time-course that depends on cAMP concentration: while the probe dissociates within few seconds in response to $2 \mu \mathrm{M}$ cAMP, the dissociation takes more than $100 \mathrm{sec}$ at $200 \mathrm{nM}$ concentration (Nikolaev et al., 2004). The PKA- 
based probe FlCRhR was nonetheless fast enough to report dynamic events such as signal propagation in dendrites of invertebrate neurons (Bacskai et al., 1993; Hempel et al., 1996) or cAMP transients in Xenopus embryonic neurons (Gorbunova and Spitzer, 2002). It should be pointed out that while PKA sensors may report changes at low cAMP concentrations with a notable delay, they nevertheless are true indicators of PKA activation, which in itself is important information.

Direct comparison of different probes was performed in retinal ganglion neurons. It appeared that the signals reported by the PKA-based probe were much slower than those recorded with ICUE2 and AKAR2.2 (Dunn et al., 2006). At first glance, it may be surprising that AKAR2.2 responded more quickly than the PKA-based probe, since PKA is responsible for the FRET change of AKAR2.2. The faster kinetics of AKAR2.2 is likely due to an "amplification" effect as one active PKA molecule is able to phosphorylate multiple AKAR2.2 molecules, producing a signal which is detected before a detectable fraction of PKA is dissociated. Epac1-camps responds in less than a few seconds in vitro, which was fast enough to report, in cultured hippocampal neurons, the diffusion of cAMP in the cytosol in response to isoprenaline (Nikolaev et al., 2004). ICUE2 also reported fast signals in rat retinal ganglion neurons (Dunn et al., 2006). These probes are still unique tools in directly reporting dynamic events occurring in the cAMP/PKA cascade at the level of individual neurons.

These kinetic methods should also allow one to further dissect the events occurring within the cascade. In retinal ganglion cells, spontaneous cAMP transients were measured with ICUE-2 with an average onset time-constants of 14 s (Dunn et al., 2006). In the same preparation, the PKA-sensitive probe AKAR2.2 also reported these fast spontaneous oscillations, although with a slightly slower onset time-constant of $20 \mathrm{~s}$ (Dunn et al., 2006). This indicates that the delay between cAMP production and phosphorylation of a PKA substrate is on the order of $6 \mathrm{~s}$, when measured in average over the whole cell. In cardiomyocytes, AKAR2 phosphorylation was monitored in response either to flash-photolysis of caged cAMP or stimulation of membrane receptors, showing a $t_{1 / 2}$ of $5 \mathrm{~s}$ and $33 \mathrm{~s}$ respectively (Saucerman et al., 2006). Similar values were obtained in intralaminar thalamic neurons, where flash photolysis of caged cAMP increased the AKAR2 ratio within few seconds, faster than the speed of the imaging system 
(Gervasi et al., 2007). On the same neurons, the AKAR2 response to the activation of $5-\mathrm{HT}_{7}$ receptors took $\sim 2.5 \mathrm{~min}$ to reach $90 \%$ of the full signal, demonstrating a fairly long delay between the binding of a neuromodulator to a membrane receptor and the transduction of a signal in the cytosol.

The decay of the cAMP/PKA response can also be studied using these sensors. In cortical neurons, dephosphorylation of AKAR2 took 1h (Gervasi et al., 2007). In contrast, spontaneous ratio oscillations were detected in retinal ganglion cells using ICUE2 or AKAR2.2, with decreases within $\sim 1 \mathrm{~min}$, indicating that cAMP unbinds from ICUE2 and is degraded by phosphodiesterases while phosphatases dephosphorylate AKAR2.2 almost as rapidly (Dunn et al., 2006). This confirms that the cAMP/PKA cascade is a highly dynamic signaling pathway in retinal ganglion neurons where phosphorylation and dephosphorylation are very active processes. This faster apparent dynamics in retinal ganglion cells than in pyramidal cortical neurons can be related to intrinsic differences in the cascade dynamics between these two neuronal types. The A206K mutations introduced in both fluorophores in AKAR2.2 also helps phosphatases access the phosphorylation site of the probe, although in cardiomyocytes, AKAR2 also seemed to dephosphorylate within few minutes (Saucerman et al., 2006).

\section{Spatial information}

The subcellular localization of intracellular second messenger signals is particularly important in the case of neuronal cells which exhibit distinct compartments with highly specialized functions. Biochemical approaches have revealed that the enzymes involved in signaling are often organized in functional microdomains, and it is a tantalizing to directly observe intracellular signals at the level of specific cellular compartments or within microcompartments.

Most recordings from neurons using genetically-encoded probes were collected in wide-field imaging with low-noise CCD cameras. Better optical sectioning would be achieved with confocal microscopy using a violet laser as shown in Drosophila brains (Shafer et al., 2008). Better penetration would be achieved in mammalian brain slices with two photon confocal imaging. This has been performed with a calcium-sensitive probe using the CFP-YFP FRET pair (Heim et al., 2007). Multiphoton imaging should provide information about cyclic nucleotide signaling in small compartments such as dendritic branches, 
spines and axon terminals in the complex and dispersive environment of a brain slice. While the optical resolution of current systems can be excellent, one should nevertheless keep in mind that cytosolic probes can diffuse and thus blur a compartmented signal. Indeed, fluorescence recovery after photobleaching (FRAP) experiments with HCN2-camps probe showed a diffusion time-course in the order of seconds within the cytosol (Nikolaev et al., 2006a). It can be expected that other freely-diffusing biosensors with similar sizes, as well as the endogenous targets of the cascade, may also diffuse from the cytosol to local signaling "microdomains". This point could be investigated further by adding to the probes attachment domains to non-diffusible cytosolic structures and comparing the kinetics of the response with those obtained with small diffusible probes.

Other subcellular compartments can also be selectively investigated using the cell's ability to carry the probe into the compartment of interest. The NLS signal sequence was used to target AKAR2 to the nucleus, which appeared strongly labeled while no fluorescence was detected in the cytosol. This nuclear probe showed that phosphorylation events also occurred in the nucleus in response to the activation of receptors at the membrane, but with much slower kinetics than in the cytosol (13 vs $2.5 \mathrm{~min}$ ) (Gervasi et al., 2007). PKA activation was also monitored at the membrane using a PKA-sensitive potassium current; activation of the same receptors produced a maximal membrane response in $0.5 \mathrm{~min}$. These results show that the membrane, the cytosol and the nucleus of neurons are separate compartments which differentially integrate the same neuromodulatory signal with their own specific time-courses. This observation is consistent with the hypothesis that receptors and enzymes of the cascade are compartmented in signaling microdomains located at the membrane close to target membrane channels, while cAMP/PKA signaling in the cytosol appears more 'diffuse'. To further confirm this model, other targeting sequences should be used to target the AKAR2 probe to such signaling microdomains, using anchoring sequences to AKAPs. While this approach proved very powerful in cell lines (Zhang et al., 2001) it has not been tested in neurons.

Heterogeneous distribution of the probe inside the cell may also affect the recorded signal. This was illustrated with cardiocytes where large molecules of the size of biosensors distribute in a striated pattern, probably as a result of "size exclusion" from the more dense myosin bands (Goaillard et al., 2001). As a result, 
ratio measurements performed on regions with different fluorescence intensities are differently affected by errors in background subtraction (see above) and comparative measurements may lead to erroneous conclusions.

At the tissue level, imaging methods are also powerful tools for providing simultaneously information from many individual cells, thereby opening the possibility to screen large number of neurons for responses to neuromodulators. This approach was used in Drosophila, where the FRET signal from geneticallydriven expression of Epac1-camps allowed the authors to compare the effect of various neuropeptides on cAMP levels in identified neurons and to show that a large population of neurons involved in the circadian rhythm of the fly respond to the peptide "pigment dispersing factor" (Shafer et al., 2008). A similar approach is applicable to cortical rat brain slices to screen for positive PKA responses in a large numbers of neurons (figure 4). Various neuromodulators were bathapplied and washed-out, while the individual responses of more than 40 neurons were recorded simultaneously with a 10x objective. After the experiment, the slice was examined with a $60 x$ objective to identify pyramidal neurons from their typical morphology (traces shown in red on figure 4), and neurons in deep cortical layers. We observed that the deep cortical neurons exhibited a larger response to serotonin than pyramidal neurons (figure 4 , blue trace and traces below). The slice was then fixed and processed for NeuN immunohistochemistry, which showed that each cell expressing AKAR2 and recorded in this experiment was a neuron. Indeed, other antibodies could be used to reveal key molecular determinants of the neuronal type and thus screen how identified neuronal types respond to series of neuromodulators.

\section{Perspectives}

Fast electrical signal transmission in the brain has long been studied using electrophysiological tools, and our knowledge of the functional integration of fast neuronal signals has made considerable progresses during the second half of the last century. The development of bioluminescent and fluorescent calcium indicators has considerably broadened this knowledge by providing spatial information from the subcellular to the network levels. In contrast, 
neuromodulatory processes lacked a direct measurement method, as the analysis of these processes mostly relied, on the one hand, on biochemical studies, and on the other hand on pharmacological and genetic methods evaluated in vivo. The huge gap between these two levels of analysis is now being filled by a novel experimental approach which uses genetically encoded biosensors to directly monitor specific second messenger responses to neuromodulatory events ex vivo and even in the living animal. Indeed, specific expression of a probe in a determined neuron type is now routine genetics in mice, and series of transgenic animals expressing various sensors in diverse brain regions will be created in the next few years. Signaling events will then be recorded directly in brain slices, from the surface of the brain using multiphoton confocal microscopy, or even from deep brain regions using fibered fluorescence microscopy (Vincent et al., 2006). This opens the possibility to reconcile the cellular data obtained from ex vivo preparations with the integrated data recorded in the physiological context of the living animal. Such an approach will undoubtedly shed new light on the integrative processes carried on in the brain by neuromodulators, and will help elucidate the mechanisms of neuromodulatory integration and its interaction with fast neuronal processing.

\section{Acknoledgements}

We would like to thank Pr Ron Harris-Warrick for critically reading this manuscript. This work was supported by CNRS, UPMC, "Fondation pour la Recherche Médicale" and "Fondation pour la Recherche sur le Cerveau" (to P. V.); and by NIH (DK073368 and CA122673), the American Heart Association, the Young Clinical Scientist Award Program of the Flight Attendant Medical Research Institute, and 3M (to J. Z.).

\section{References:}

Adams, S.R. et al. (1991) Fluorescence ratio imaging of cyclic AMP in single cells. Nature, 349, 694-697.

Allen, M.D. \& Zhang, J. (2006) Subcellular dynamics of protein kinase A activity visualized by FRET-based reporters. Biochemical and Biophysical Research Communications, 348, 716-721.

Bacskai, B.J. et al. (1993) Spatially resolved dynamics of cAMP and protein kinase A subunits in Aplysia sensory neurons. Science, 260, 222-226. 
DiPilato, L.M., Cheng, X. \& Zhang, J. (2004) Fluorescent indicators of cAMP and Epac activation reveal differential dynamics of cAMP signaling within discrete subcellular compartments. Proceedings of the National Academy of Sciences of the United States of America, 101, 16513-16518.

Domingo, B. et al. (2007) Imaging FRET standards by steady-state fluorescence and lifetime methods. Microsc Res Tech,

Dunn, T.A. et al. (2006) Imaging of cAMP levels and protein kinase a activity reveals that retinal waves drive oscillations in second-messenger cascades. Journal of Neuroscience, 26, 12807-12815.

Gervasi, N. et al. (2007) Dynamics of PKA Signaling at the Membrane, in the Cytosol and in the Nucleus of Neurons in Mouse Brain Slices. Journal of Neuroscience, 27, 2744-2750.

Gibbs, C.S. et al. (1992) Systematic mutational analysis of cAMP-dependent protein kinase identifies unregulated catalytic subunits and defines regions important for the recognition of the regulatory subunit. Journal of Biological Chemistry, 267, 4806-4814.

Goaillard, J.-M., Vincent, P. \& Fischmeister, R. (2001) Simultaneous measurements of intracellular cAMP and L-type Ca2+ current in single frog ventricular myocytes. Journal of Physiology, 530, 79-91. Gorbunova, Y.V. \& Spitzer, N.C. (2002) Dynamic interactions of cyclic AMP transients and spontaneous $\mathrm{Ca}(2+)$ spikes. Nature, 418, 93-96. Gordon, G.W. et al. (1998) Quantitative fluorescence resonance energy transfer measurements using fluorescence microscopy. Biophysical Journal, 74, 27022713.

Grynkiewicz, G., Poenie, M. \& Tsien, R.Y. (1985) A new generation of Ca2+ indicators with greatly improved fluorescence properties. Journal of Biological Chemistry, 260, 3440-3450.

$\mathrm{Gu}$, Y. et al. (2004) Quantitative fluorescence resonance energy transfer (FRET) measurement with acceptor photobleaching and spectral unmixing. Journal of Microscopy, 215, 162-173.

Heim, N. et al. (2007) Improved calcium imaging in transgenic mice expressing a troponin C-based biosensor. Nat Methods, 4, 127-129.

Hempel, C.M. et al. (1996) Spatio-temporal dynamics of cAMP signals in an intact neural circuit. Nature, 384, 166-169. 
Hepp, R. et al. (2007) Phosphodiesterase type 2 and the homeostasis of cyclic GMP in living thalamic neurons. Journal of Neurochemistry, 102, 1875-1886. Honda, A. et al. (2005) Cygnets: in vivo characterization of novel cGMP indicators and in vivo imaging of intracellular cGMP. Methods in Molecular Biology, 307, 27-43.

Honda, A. et al. (2001) Spatiotemporal dynamics of guanosine 3',5'-cyclic monophosphate revealed by a genetically encoded, fluorescent indicator. Proceedings of the National Academy of Sciences of the United States of America, 98, 2437-2442.

Hoppe, A., Christensen, K. \& Swanson, J.A. (2002) Fluorescence resonance energy transfer-based stoichiometry in living cells. Biophysical Journal, 83, 36523664.

Houslay, M.D. \& Milligan, G. (1997) Tailoring cAMP-signalling responses through isoform multiplicity. Trends in Biochemical Sciences, 22, 217-224. Kaupp, U.B. \& Seifert, R. (2001) Molecular Diversity of Pacemaker Ion Channels. Annu. Rev. Physiol, 63, 235-257.

Kemp, B.E. (1980) Phosphorylation of acyl and dansyl derivatives of the peptide Leu-Arg-Arg-Ala-Ser-Leu-Gly by the cAMP-dependent protein kinase. Journal of Biological Chemistry, 255, 2914-2918.

Lissandron, V. et al. (2007) Transgenic fruit-flies expressing a FRET-based sensor for in vivo imaging of cAMP dynamics. Cellular Signalling, 19, 22962303.

Lissandron, V. et al. (2005) Improvement of a FRET-based indicator for cAMP by linker design and stabilization of donor-acceptor interaction. Journal of Molecular Biology, 354, 546-555.

Lohse, M.J. et al. (2008) Optical techniques to analyze real-time activation and signaling of G-protein-coupled receptors. Trends Pharmacol Sci, 29, 159-165. Mongillo, M. et al. (2004) Fluorescence resonance energy transfer-based analysis of cAMP dynamics in live neonatal rat cardiac myocytes reveals distinct functions of compartmentalized phosphodiesterases. Circulation Research, 95, 67-75. Namiki, S. et al. (2005) NO signalling decodes frequency of neuronal activity and generates synapse-specific plasticity in mouse cerebellum. Journal of Physiology, 566, 849-863. 
Nausch, L.W. et al. (2008) Differential patterning of cGMP in vascular smooth muscle cells revealed by single GFP-linked biosensors. Proceedings of the National Academy of Sciences of the United States of America, 105, 365-370.

Nikolaev, V.O. et al. (2004) Novel single chain cAMP sensors for receptorinduced signal propagation. Journal of Biological Chemistry, 279, 3721537218.

Nikolaev, V.O. et al. (2006a) Cyclic AMP Imaging in Adult Cardiac Myocytes Reveals Far-Reaching \{beta\}1-Adrenergic but Locally Confined \{beta\}2Adrenergic Receptor-Mediated Signaling. Circulation Research, 99, 1084-1091. Nikolaev, V.O., Gambaryan, S. \& Lohse, M.J. (2006b) Fluorescent sensors for rapid monitoring of intracellular cGMP. Nature Methods, 3, 23-25.

Ponsioen, B. et al. (2004) Detecting cAMP-induced Epac activation by fluorescence resonance energy transfer: Epac as a novel cAMP indicator. $E M B O$ Reports, 5, 1176-1180.

Rich, T.C. \& Karpen, J.W. (2002) Review article: cyclic AMP sensors in living cells: what signals can they actually measure? Ann Biomed Eng, 30, 1088-1099. Rich, T.C. et al. (2001a) In vivo assessment of local phosphodiesterase activity using tailored cyclic nucleotide-gated channels as cAMP sensors. Journal of General Physiology, 118, 63-78.

Rich, T.C. et al. (2001b) A uniform extracellular stimulus triggers distinct cAMP signals in different compartments of a simple cell. Proceedings of the National Academy of Sciences of the United States of America, 98, 13049-13054.

Russwurm, M. et al. (2007) Design of fluorescence resonance energy transfer (FRET)-based cGMP indicators: a systematic approach. Biochemical Journal, 407, 69-77.

Sato, M., Hida, N. \& Umezawa, Y. (2005) Imaging the nanomolar range of nitric oxide with an amplifier-coupled fluorescent indicator in living cells. Proceedings of the National Academy of Sciences of the United States of America, Sato, M. et al. (2000) Fluorescent indicators for cyclic GMP based on cyclic GMP-dependent protein kinase Ialpha and green fluorescent proteins. Analytical Chemistry, 72, 5918-5924. 
Saucerman, J.J. et al. (2006) Systems analysis of PKA-mediated phosphorylation gradients in live cardiac myocytes. Proceedings of the National Academy of Sciences of the United States of America, 19, 2650-2658.

Shafer, O.T. et al. (2008) Widespread Receptivity to Neuropeptide PDF throughout the Neuronal Circadian Clock Network of Drosophila Revealed by Real-Time Cyclic AMP Imaging. Neuron, 58, 161-163.

Smith, C.M. et al. (1999) The catalytic subunit of cAMP-dependent protein kinase: prototype for an extended network of communication. Prog Biophys Mol Biol, 71, 313-341.

Tsien, R.Y. \& Harootunian, A.T. (1990) Practical design criteria for a dynamic ratio imaging system. Cell Calcium, 11, 93-109.

Vincent, P. et al. (2006) Live imaging of neural structure and function by fibred fluorescence microscopy. EMBO Reports, 7, 1154-1161.

Vincent, P. \& Brusciano, D. (2001) Cyclic AMP imaging in neurones in brain slice preparations. Journal of Neuroscience Methods, 108, 189-198. Violin, J.D. et al. (2008) 32 -Adrenergic Receptor Signaling and Desensitization Elucidated by Quantitative Modeling of Real Time cAMP Dynamics. Journal of Biological Chemistry, 283, 2949-2961.

Willoughby, D. \& Cooper, D.M. (2008) Live-cell imaging of cAMP dynamics. Nature Methods, 5, 29-36.

Zaccolo, M. et al. (2000) A genetically encoded, fluorescent indicator for cyclic AMP in living cells. Nature Cell Biology, 2, 25-29.

Zacharias, D.A. et al. (2002) Partitioning of lipid-modified monomeric GFPs into membrane microdomains of live cells. Science, 296, 913-916.

Zetterqvist, O. et al. (1976) The minimum substrate of cyclic AMP-stimulated protein kinase, as studied by synthetic peptides representing the phosphorylatable site of pyruvate kinase (type L) of rat liver. Biochemical and Biophysical Research Communications, 70, 696-703.

Zhang, J. et al. (2005) Insulin disrupts beta-adrenergic signalling to protein kinase A in adipocytes. Nature, 437, 569-573.

Zhang, J. et al. (2001) Genetically encoded reporters of protein kinase A activity reveal impact of substrate tethering. Proceedings of the National Academy of Sciences of the United States of America, 98, 14997-15002. 
Zimmermann, T. et al. (2002) Spectral imaging and linear un-mixing enables improved FRET efficiency with a novel GFP2-YFP FRET pair. FEBS Letters, 531, 245-249.

\section{Figure legends}

Table 1: biosensors for cyclic nucleotides.

Figure 1: schematic representation of probes for cyclic nucleotide signaling. A: ICUE2 (and other Epac-derived cAMP-sensitive probes) contains one cAMP-binding site from Epac. Binding of one cAMP molecule induces a conformational change that decreases FRET. B: AKAR2 contains a PKA consensus phosphorylation site and a FHA domain. When the threonine is phosphorylated by PKA, the FHA domain binds to it, increasing FRET. For all probes, the conformational change is reversible upon cyclic nucleotide unbinding or phosphatase-mediated dephosphorylation. C: Cygnet2 is constituted of the regulatory and catalytic domains of PKG with CFP and YFP fused at $\mathrm{N}$ - and C-terminals respectively. Binding of two cGMP to the regulatory subunit induce a conformational change which results in a FRET decrease.

Figure 2: Expression of the probe does not affect the firing properties of neurons in brain slices. Left: image combining transillumination and epifluorescence. Right: current-clamp recording of the same neuron upon depolarizing and hyperpolarizing current injection. A: a thalamocortical neuron in the ventrobasal complex displays the characteristic tonic firing upon depolarization. Hyperpolarization induces the 'sag', followed by low-threshold calcium spike upon repolarization. B: a layer $\mathrm{V}$ pyramidal cortical neuron fires tonically upon depolarization.

Figure 3: Blocking phosphodiesterases increases PKA activity. A thalamocortical neuron in the ventrobasal complex in a thalamic brain slice is recorded with the AKAR2 probe. IBMX strongly increases the F535/F480 fluorescence ratio, indicating a strong activation of PKA that is not further increased by subsequent forskolin application. Top: YFP fluorescence image (left) and pseudocolor images in control (middle) and during bath application of IBMX (right). Calibration squares indicate the intensity range (from left to right) in counts/pixel/s and the ratio range (from bottom to top). The size of the square is $10 \mu \mathrm{m}$. The graph shows the average ratio measured over the cell during the experiment.

Figure 4: Cortical brain slice expressing AKAR2 and recorded with a 10x objective. A: Pseudocolor images in control (top) and during dopamine application (bottom). B: 40 neurons were analyzed for their F535/F480 ratio change during bath application of noradrenaline, dopamine, 5-HT and forskolin. After the recording, at least 9 cells (red traces) were unambiguously identified as pyramidal neurons from their morphology. Other cells (blue trace and below) were in layer VI and were not pyramidal neurons. C: the slice was fixed and the same neurons were examined with a 60x objective for AKAR fluorescence (top image) and NeuN immunoreactivity (bottom image). 
A: ICUE2

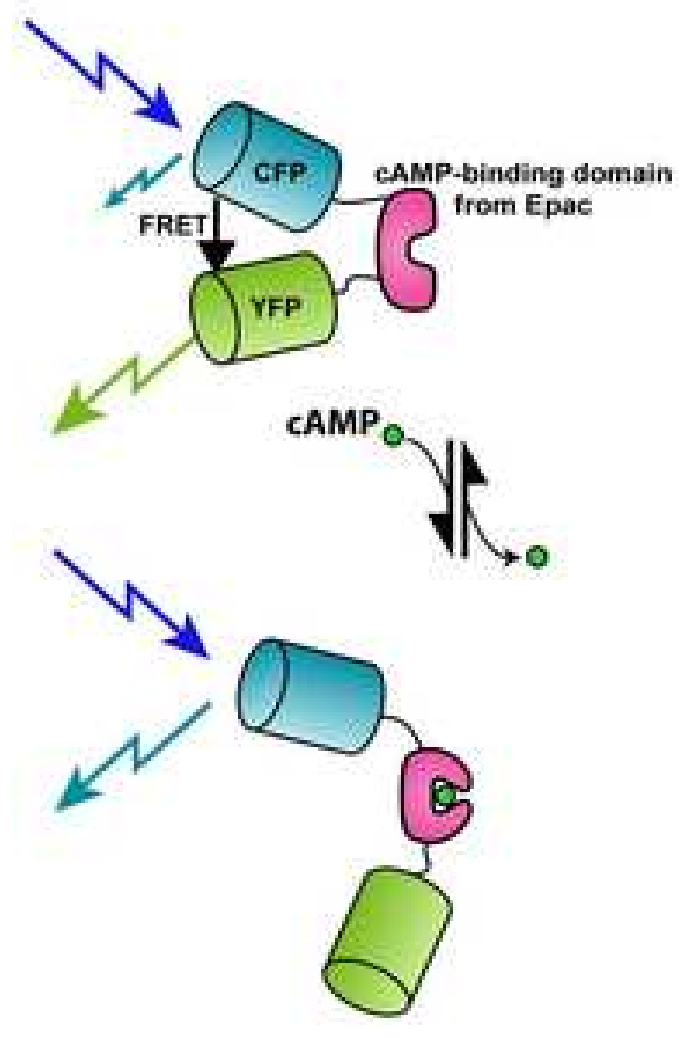

B: AKAR2
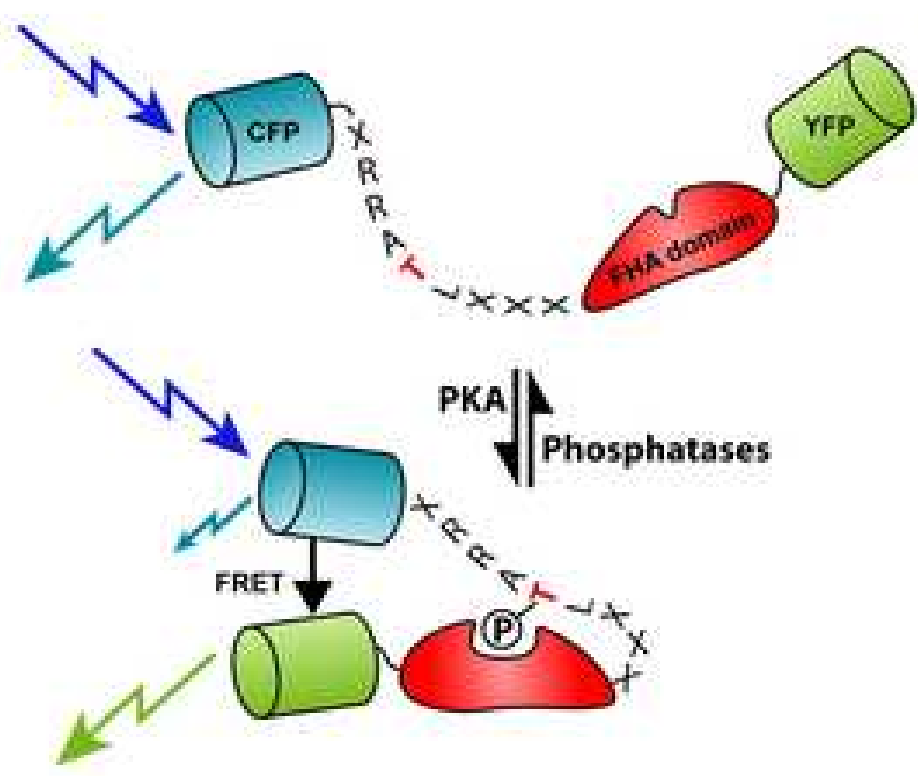

C: Cygnet 2

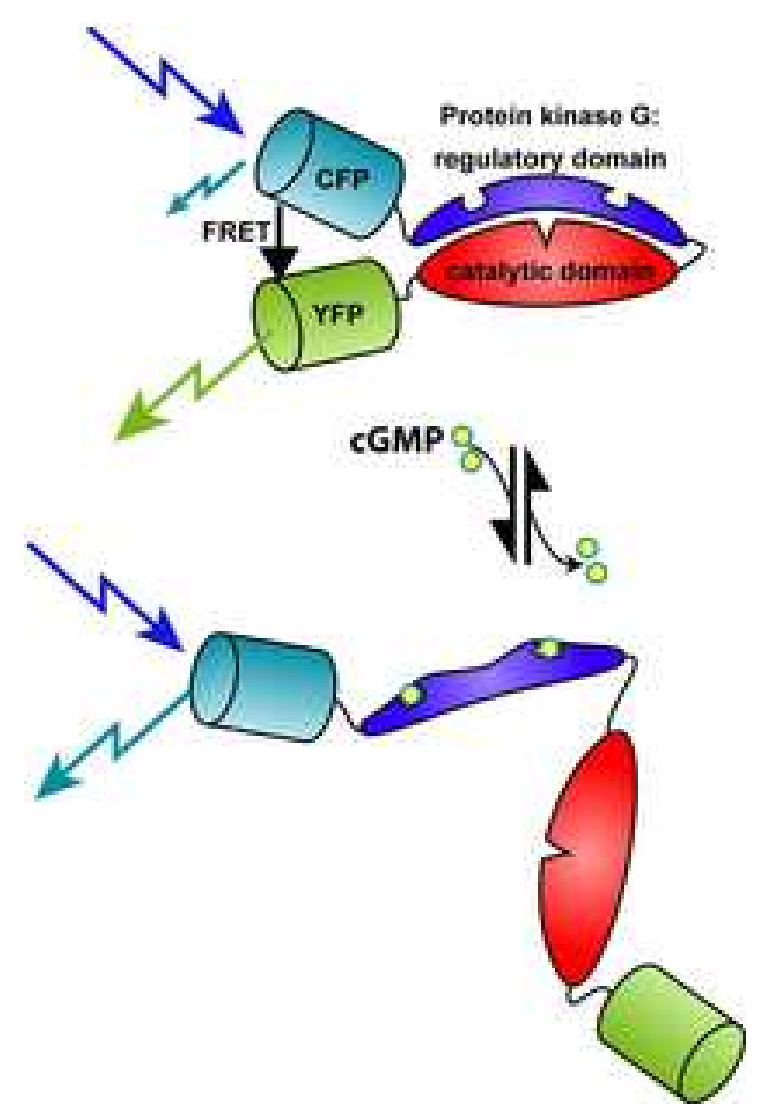


A

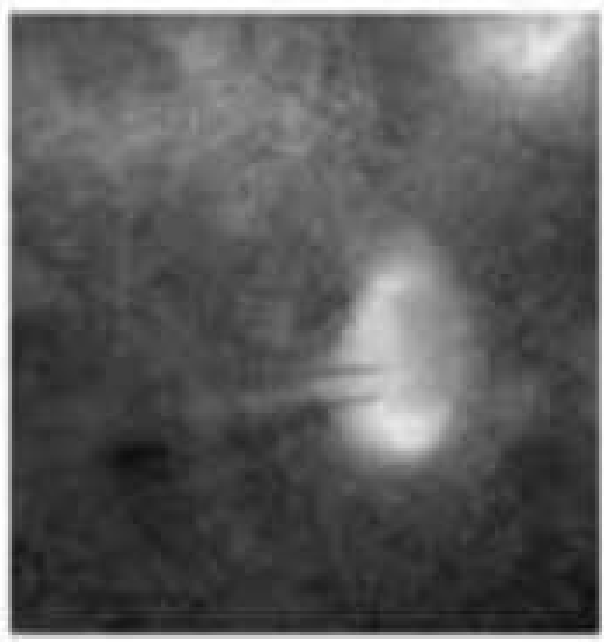

B

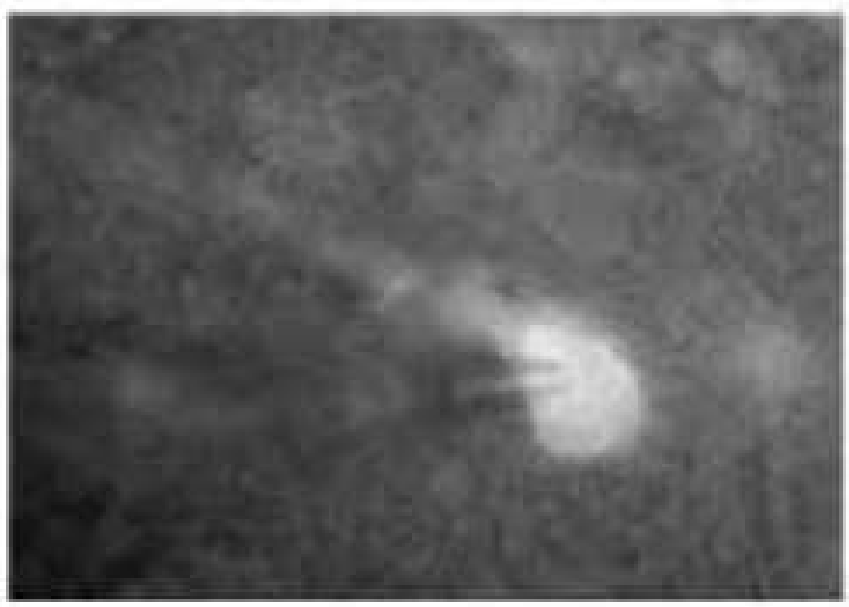

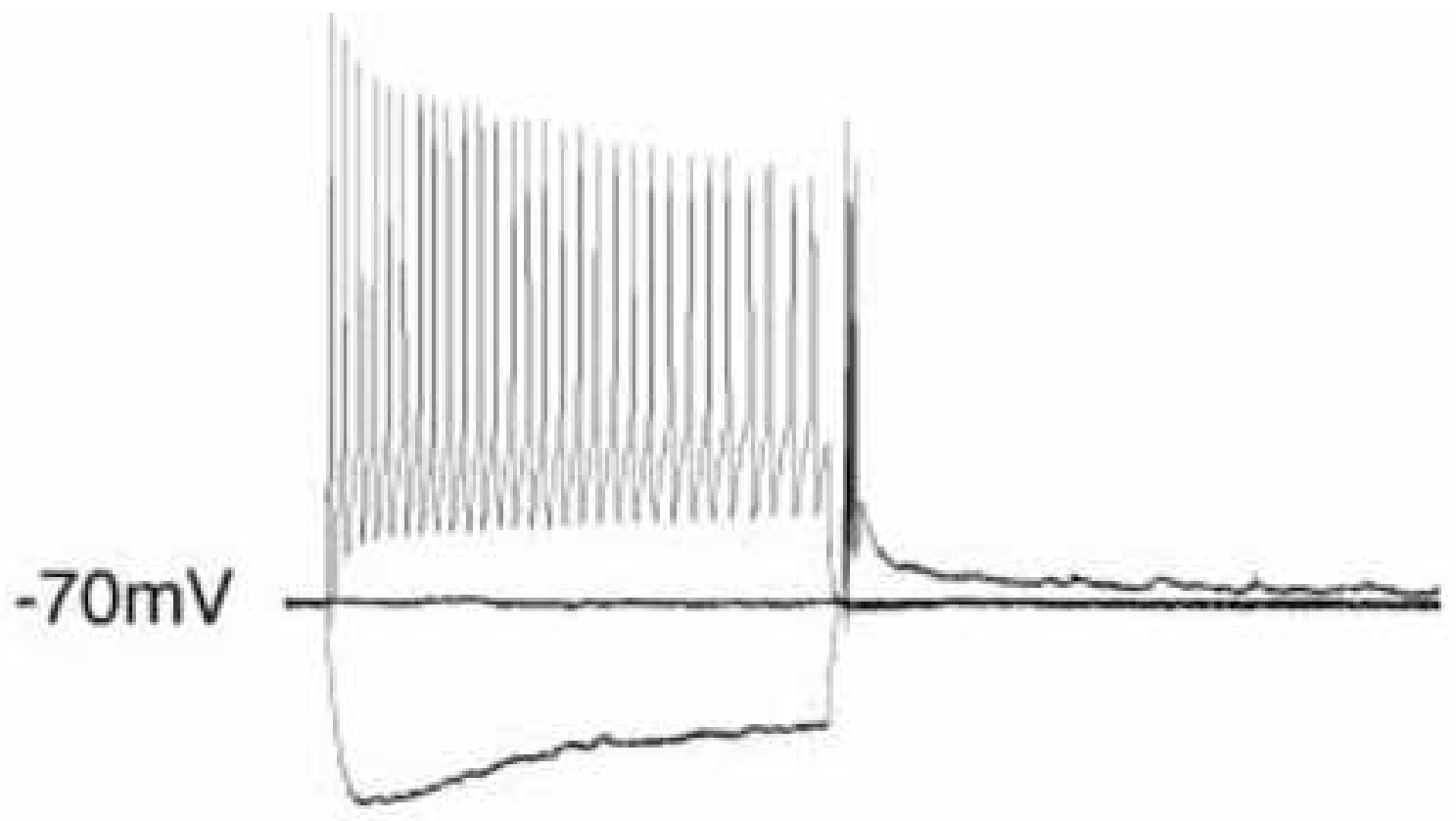

$\frac{\mathrm{J}}{200 \mathrm{~ms}} \mathrm{mV}$

$-70 \mathrm{mV}$
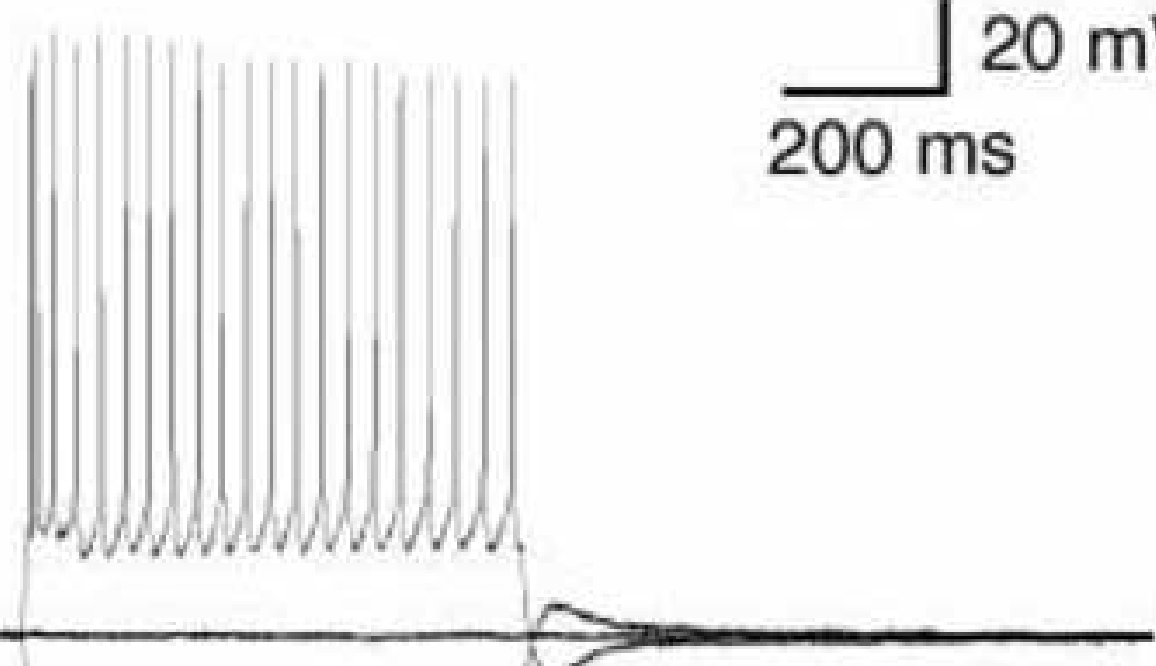
Click here to download high resolution image
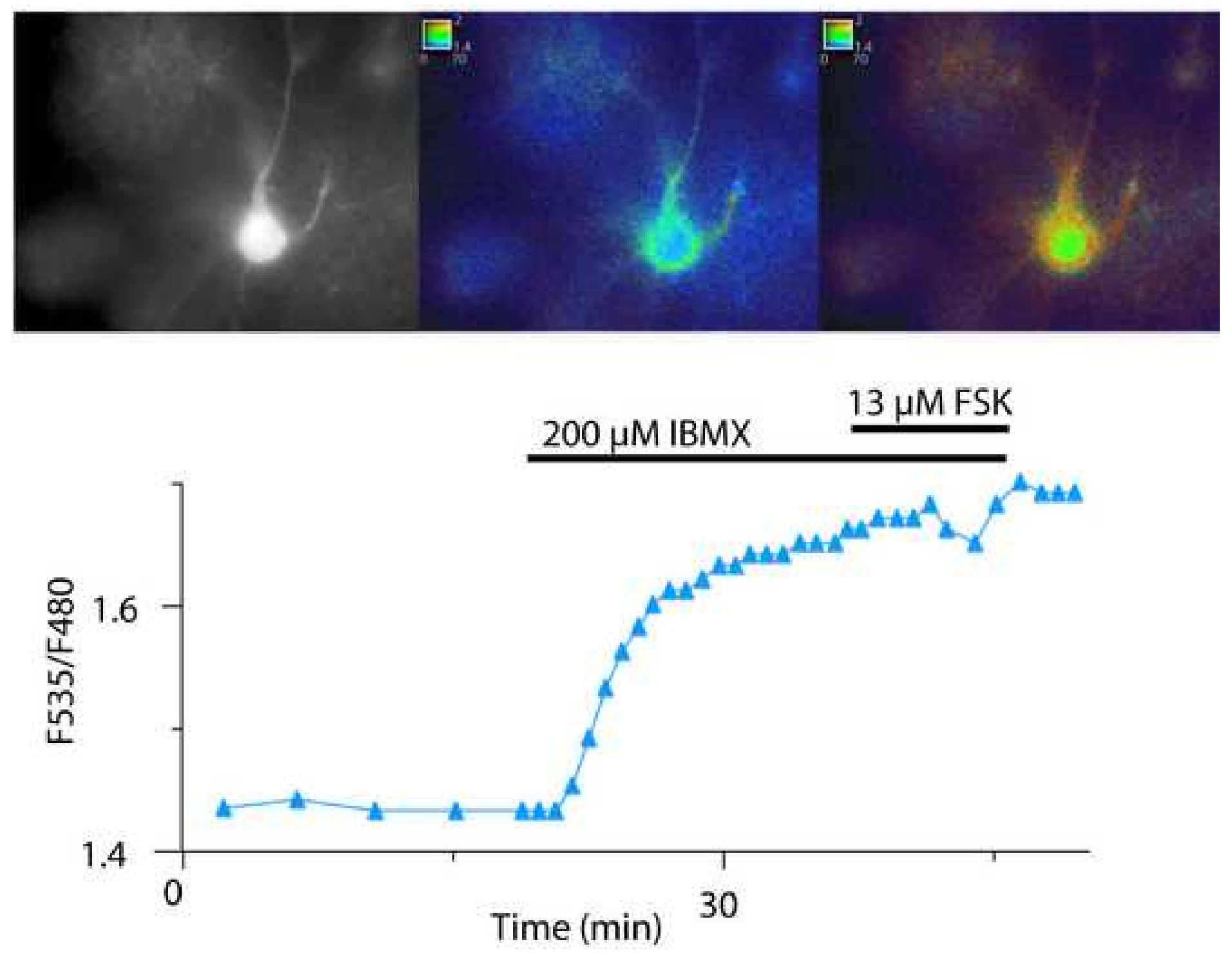
Click here to download high resolution image

A

B

noradrenaline dopamine 5-HT forskolin
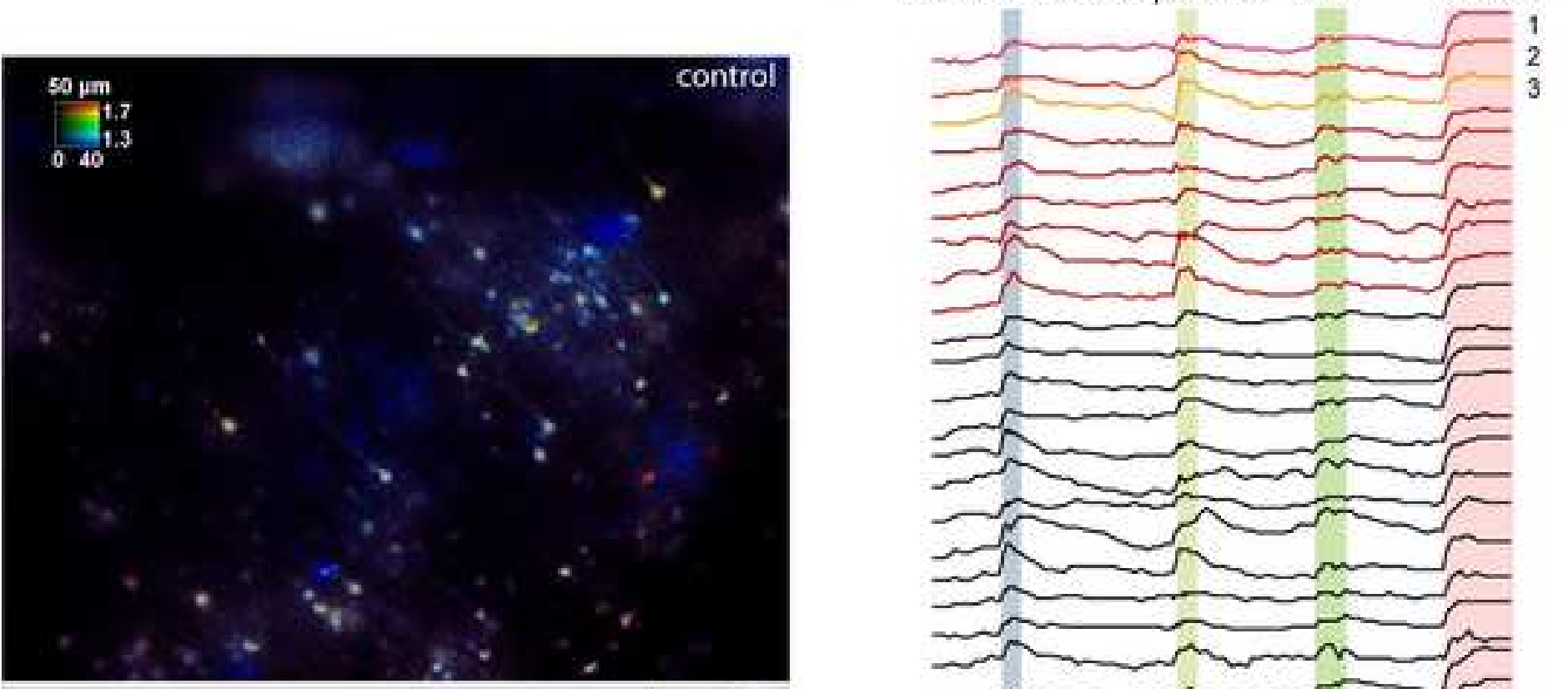

C

dopamine
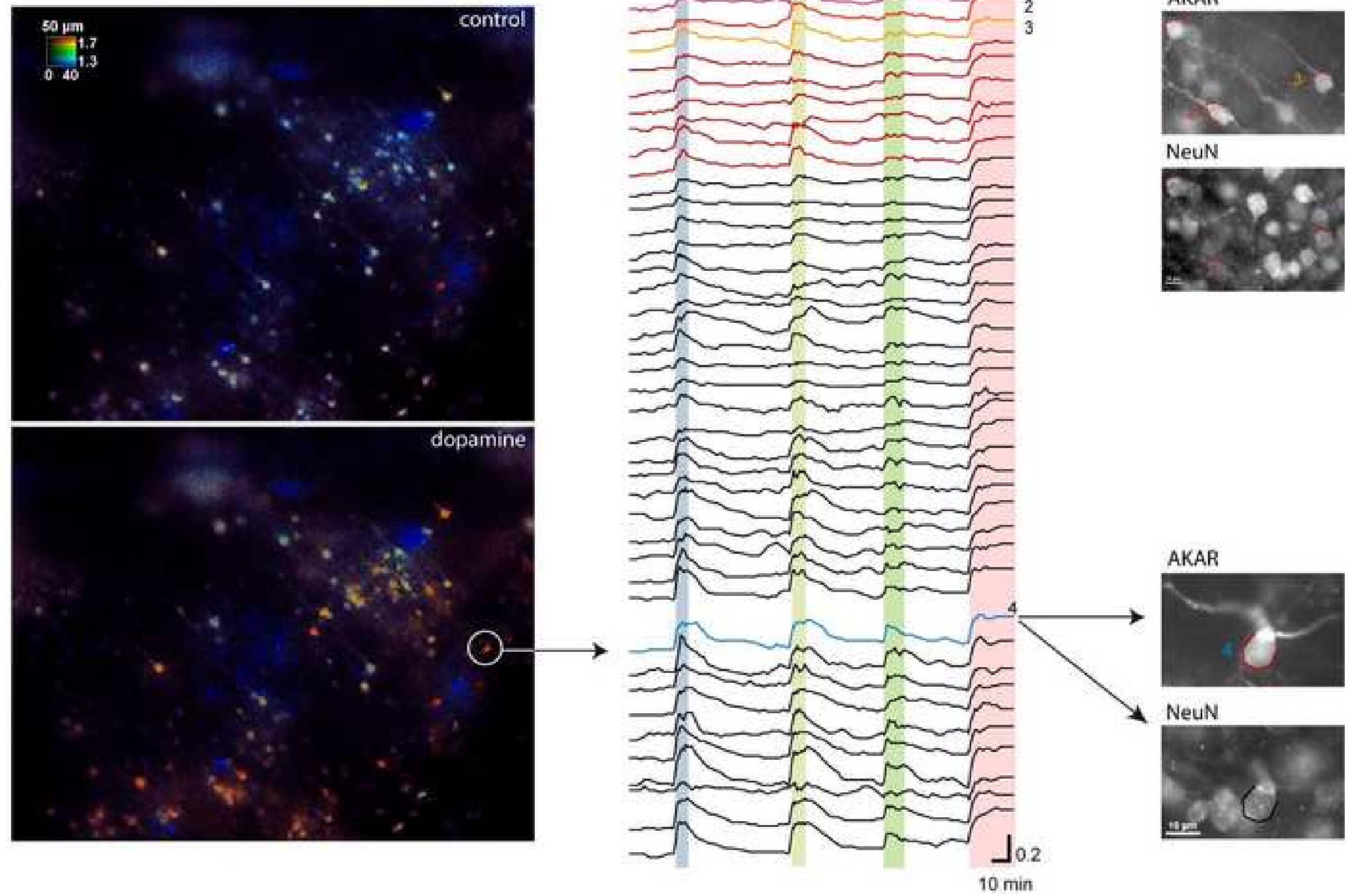

4 NeuN

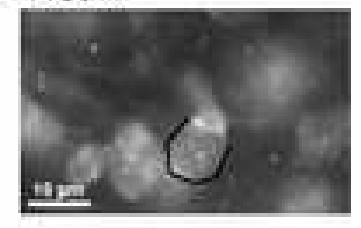

